\title{
Relations between Plasma von Willebrand Factor or Endothelin-1 and Restenosis following Carotid Artery Stenting
}

\author{
Hua Yang ${ }^{a}$ Jian-Xin Xu ${ }^{b}$ Xiang-Zhi Kong ${ }^{a}$ Zeng-Guang Ren ${ }^{a}$ \\ Zhang-Yong Xia ${ }^{b}$ Huai-Qian Qu ${ }^{b}$ Le-Xin Wang ${ }^{c, d}$ \\ ${ }^{a}$ Department of Neurology, the Third People's Hospital of Liaocheng, and Departments of ${ }^{\text {b Neurology and }}$ \\ 'Cardiology, Liaocheng People's Hospital and Liaocheng Clinical School of Taishan Medical University, Liaocheng, \\ PR China; ${ }^{d}$ School of Biomedical Sciences, Charles Sturt University, Wagga Wagga, N.S.W., Australia
}

\section{Key Words}

Carotid artery stenosis $\cdot$ Stenting $\cdot$ Von Willebrand factor •

Endothelin-1 · Stroke

\begin{abstract}
Objective: This study was designed to investigate the relation between plasma von Willebrand factor (VWF) or endothelin-1 (ET-1) and post-carotid artery stenting (CAS) restenosis. Materials and Methods: Plasma levels of VWF and ET-1 were measured in 61 patients ( 36 males, mean age 64.4 \pm 6.8 years) before and after CAS. The mean follow-up time was $13.8 \pm 1.7$ months (range, 6-63). In-stent restenosis was defined as a $>10 \%$ narrowing of the vascular lumen with or without ischemic symptoms following CAS. Results: In-stent restenosis was identified in 14 (23\%) patients, including 3 with $>50 \%$ restenosis. In the restenosis group, mean VWF and ET-1 levels at 2 weeks, 1 and 6 months after CAS were higher than the baseline levels $(p<0.05$ or $p<0.01)$. Mean levels of VWF and ET-1 in the restenosis group were higher than in the non-restenosis group within 6 months after CAS $(p<0.05$ or $p<0.01)$. Conclusion: Persistent elevation in plasma VWF and ET-1 within the first 6 months of CAS was found in patients with in-stent restenosis.
\end{abstract}

Copyright $\odot 2012$ S. Karger AG, Basel
(C) 2012 S. Karger AG, Basel

1011-7571/12/0216-0538\$38.00/0

Fax +4161306 1234

E-Mail karger@karger.ch

www.karger.com
Accessible online at: www.karger.com/mpp

\section{Introduction}

Carotid artery stenosis is a major cause of stroke, transient ischemic attacks or death [1-3]. Although medical therapy remains the mainstay of treatment in patients with asymptomatic stenosis $[2,3]$, invasive treatment is indicated for symptomatic patients with stenosis greater than $50 \%$, and in a select group of asymptomatic patients with severe stenosis [2]. Carotid artery stenting (CAS) has been increasingly used as an alternative treatment to carotid endarterectomy for symptomatic carotid stenosis $[4,5]$. Restenosis after CAS remains a clinical challenge and long-term safety and efficacy of CAS is yet to be elucidated [4]. Factors predicting in-stent restenosis and future need for target lesion revascularization remain undetermined [4]. Coronary angioplasty and stenting was associated with a significant increase in plasma levels of von Willebrand factor (VWF) and endothelin-1 levels [68]. Elevation of plasma VWF may contribute to the pathogenesis of acute or subacute stent thrombosis and restenosis after multiple stenting [6], but association between endothelin-1 and restenosis of the coronary artery is controversial $[7,8]$. Our recent study showed that plasma levels of VWF and endothelin-1 were elevated within the first 2 weeks of CAS, but the post-CAS VWF and endothelin-1 elevation was not related to the restenosis of carotid arteries [9]. The role of endothelial injuries and sub- 
Table 1. Comparison of baseline data and CAS results between patients with and without post-CAS restenosis

\begin{tabular}{lccl}
\hline Items & $\begin{array}{l}\text { Restenosis } \\
\text { group }(\mathrm{n}=14)\end{array}$ & $\begin{array}{l}\text { Non-restenosis } \\
\text { group }(\mathrm{n}=47)\end{array}$ & $\begin{array}{l}\mathrm{p} \\
\text { value }\end{array}$ \\
\hline Sex, male & $8(57.1 \%)$ & $25(53.2 \%)$ & 0.795 \\
Age, years & $64.89 \pm 7.12$ & $64.06 \pm 6.75$ & 0.691 \\
History of smoking & $6(42.9 \%)$ & $18(38.3 \%)$ & 0.759 \\
Hypertension & $5(35.7 \%)$ & $20(42.6 \%)$ & 0.648 \\
Diabetes & $4(28.6 \%)$ & $8(17.0 \%)$ & 0.568 \\
Dyslipidemia & $4(28.6 \%)$ & $16(34.0 \%)$ & 0.953 \\
Blood glucose, mmol/l & $7.30 \pm 2.47$ & $7.01 \pm 2.38$ & 0.693 \\
Total cholesterol, mmol/1 & $5.98 \pm 2.26$ & $6.11 \pm 2.51$ & 0.863 \\
Triglyceride, mmol/l & $2.61 \pm 0.64$ & $2.67 \pm 0.68$ & 0.770 \\
LDL, mmol/l & $3.59 \pm 0.54$ & $3.65 \pm 0.57$ & 0.728 \\
ACE or ARB & $12(85.7 \%)$ & $41(87.2 \%)$ & 0.639 \\
Severity of stenosis before CAS & & \\
$\quad>50-70 \%$ & $6(42.9 \%)$ & $26(55.3 \%)$ & 0.412 \\
$\quad>70 \%$ & $8(57.1 \%)$ & $21(44.7 \%)$ & 0.412 \\
CAS results & & & \\
$\quad$ Single stent & $7(50.0 \%)$ & $27(57.4 \%)$ & 0.622 \\
$\quad$ Two stents & $7(50.0 \%)$ & $20(42.6 \%)$ & 0.622 \\
\hline
\end{tabular}

$\mathrm{ACE}=$ Angiotensin-converting inhibitor $\mathrm{ARB}=$ angiotensin receptor blocker.

sequent changes in endothelium-derived VWF or endothelin-1 in post-CAS restenosis has not been previously reported. In this study, we investigated the relations between post-CAS restenosis and plasma levels of VWF or endothelin-1 12 months after CAS.

\section{Materials and Methods}

\section{Patient Selection}

This study was approved by the Institutional Review Committee of our hospital and adhered to the principles of the Declaration of Helsinki. Written informed consent was obtained from all study participants. Between February 2006 and January 2009, 67 patients (36 males, mean age $64.4 \pm 6.8$ years; range, $56-73$ ) who had a history of transient ischemic attack and met the selection criteria for CAS were selected for this study. Five patients who did not complete the 1-year follow-up were subsequently excluded from this study. The remaining 61 patients completed 12 months' follow-up. The indications for CAS were more than $50 \%$ of lumen stenosis accompanying clinical symptoms, or asymptomatic patients with more than 70\% lumen stenosis [9]. Computed tomographic (CT) carotid angiography was used to diagnose carotid stenosis. Exclusion criteria included severe liver or kidney diseases, autoimmune disease, hematological disorders, tumor, or recent surgeries/trauma. Patients who did not complete the 12-month follow-up study including those who died before the end of the follow-up were excluded from final analysis.

\section{CAS Procedures}

The CAS procedures used in this study have been reported previously [9]. In brief, under local anesthesia, an 8 -french guide catheter was inserted through the introducer sheath to the distal end of the common carotid artery. A protective umbrella was inserted and released to the distal part of carotid stenosis, and a bare metal stent (Acculink, Abbott, USA) was positioned on the stenotic segment of the carotid artery. The procedure was considered successful if the post-stenting carotid angiography showed $<10 \%$ stenosis. The general characteristics of patients with or without restenosis are listed in table 1 . At baseline, there was no significant difference in age, sex, blood glucose, hypertension and blood lipids between the two groups ( $p>0.05$ ). There was no statistically significant difference in the severity of carotid stenosis and in the number of stents being implanted ( $p>0.05$ ). Following CAS, all patients were given clopidogrel and aspirin as antiplatelet therapy. Twelve (85.7\%) patients in the restenosis group and $41(87.2 \%, \mathrm{p}>$ $0.05)$ in the non-restenosis group were treated with an oral angiotensin converting enzyme inhibitor or an angiotensin receptor blocker to maintain blood pressure below 120/80 $\mathrm{mm} \mathrm{Hg}$.

\section{Measurement of VWF and Endothelin-1}

Venous blood samples were obtained immediately before, and $1 \mathrm{~h}, 2$ weeks, 6 months and 12 months after CAS. The blood samples were collected into testing tubes containing $2 \%$ ethylenediaminetetraacetic acid and were centrifuged for $10 \mathrm{~min}$ at 3,000 $\mathrm{rpm}$. The supernatant plasma was separated and stored at $-20^{\circ} \mathrm{C}$ before the measurement within 4 weeks of the blood sample collection. The levels of VWF were measured by enzyme-linked immunosorbent assay (Sunlight Biotech, Fujain, China), and the level of endotheilin-1 was measured by radioimmunoassay kit (Institute of Immunology, Beijing, China). The detection procedures were performed strictly following the methods and steps instructed by the manufacturer.

\section{Follow-Up}

Patients were followed up in our hospital clinics monthly in the first 3 months, then every 2 months. Clinical examination was conducted and medications were reviewed during each visit to ensure compliance to clopidogrel, aspirin, atorvastatin and antihypertensive drugs. The CT carotid angiogram was performed at 12 months after stenting in all patients by Brilliance CT-64-channel scanner (Philips Medical Systems). The images of carotid artery were viewed separately by two senior radiologists who reviewed the CT angiography and were blinded to the blood levels of VWF or endothelin-1. In-stent restenosis was defined as a $>10 \%$ narrowing of the vascular lumen with or without ischemic symptoms following the CAS.

\section{Statistical Analysis}

Statistical analysis was performed using SPSS, version 13.0. Data from restenosis and non-restenosis groups were expressed with means \pm standard deviations. Student's t test was performed to analyze the significance of differences in endothelin-1 and VWF before and after stenting. One-way ANOVA was used to compare the differences in baseline data, VWF or endothelin-1 level between the restenosis and non-restenosis groups. Categorical data were analyzed by $\chi^{2}$ test; $\mathrm{p}<0.05$ was considered as statistically significant. 
Table 2. Changes in VWF and endothelin-1 before and after CAS in 61 patients

\begin{tabular}{|c|c|c|c|c|c|c|}
\hline & Baseline & $1 \mathrm{~h}$ & 2 weeks & 1 month & 6 months & 12 months \\
\hline VWF, \% & $108.1 \pm 11.5$ & $117.5 \pm 12.5^{* *}$ & $110.7 \pm 11.6$ & $109.3 \pm 11.0$ & $114.1 \pm 12.1^{* *}$ & $107.9 \pm 11.9$ \\
\hline Endothelin-1 & $72.10 \pm 5.64$ & $77.3 \pm 6.6^{* *}$ & $74.7 \pm 6.5^{*}$ & $72.9 \pm 5.4$ & $74.9 \pm 6.7^{*}$ & $72.6 \pm 6.8$ \\
\hline
\end{tabular}

Compared to those before CAS, ${ }^{*} \mathrm{p}<0.05 ;{ }^{* *} \mathrm{p}<0.01$.

Table 3. Comparison of VWF and endothelin-1 (ET-1) between restenosis and non-restenosis groups

\begin{tabular}{|c|c|c|c|c|c|c|c|}
\hline \multirow[t]{2}{*}{ Item } & \multirow[t]{2}{*}{ Group } & \multirow[t]{2}{*}{ Before } & \multicolumn{5}{|l|}{ After stenting } \\
\hline & & & $1 \mathrm{~h}$ & 2 weeks & 1 month & 6 months & 12 months \\
\hline \multirow[t]{2}{*}{ VWF, \% } & non-restenosis & $108.0 \pm 11.4$ & $113.1 \pm 11.5^{\circ}$ & $108.5 \pm 11.9$ & $108.4 \pm 12.0$ & $112.0 \pm 11.8$ & $107.7 \pm 11.8$ \\
\hline & restenosis & $108.6 \pm 11.8$ & $128.9 \pm 11.8^{* *}$ & $118.2 \pm 12.3^{*}$ & $112.3 \pm 12.0^{*}$ & $121.6 \pm 12.1^{* *}$ & $108.9 \pm 12.0$ \\
\hline \multirow[t]{2}{*}{ ET-1, ng/l } & non-restenosis & $71.9 \pm 5.6$ & $76.3 \pm 6.4^{\circ}$ & $73.9 \pm 5.7$ & $72.2 \pm 5.1$ & $73.8 \pm 5.8$ & $72.2 \pm 6.7$ \\
\hline & restenosis & $72.8 \pm 5.8$ & $80.9 \pm 7.1^{* *, o}$ & $77.5 \pm 5.9^{*, o}$ & $75.6 \pm 6.9^{*, o}$ & $78.4 \pm 7.0^{*, \mathrm{o}}$ & $73.7 \pm 7.1$ \\
\hline
\end{tabular}

Compared to those in the non-restenosis group, ${ }^{*} \mathrm{p}<0.05 ;{ }^{*} \mathrm{p}<0.01 ;{ }^{\circ} \mathrm{p}<0.01$ compared with baseline value within the same group.

\section{Results}

\section{Post-CAS Restenosis}

All patients were symptomatic before the CAS, which was successful in all patients with a dilation of the stenotic segment of the carotid artery of more than $90 \%$. There were no complications such as stroke, vascular injuries or bleeding requiring blood transfusion following CAS. During the follow-up, 1 patient died of acute myocardial infarction 8 months following CAS. The mean follow-up time was $13.8 \pm 1.7$ months (range, 12-63). Restenosis was detected by CT carotid angiogram in 14 patients (23\%). In 3 patients (4.9\%), restenosis involved more than $50 \%$ of the vascular lumen. No further intervention was required in the 3 patients as they were asymptomatic. Four (6.6\%) and 7 (11.5\%) patients had restenosis of $30-50 \%$ of the vascular lumen and $11-30 \%$ of the lumen, respectively. None of these patients with restenosis had transient ischemic attack or other ischemic symptoms, therefore further intervention was not required.

\section{Changes in VWF and Endothelin-1 following CAS}

As shown in table 2, the levels of VWF and endothelin-1 of the 61 patients at $1 \mathrm{~h}$ after CAS were higher than the baseline levels. The 6-month VWF and endothelin-1 were also higher than the baseline levels ( $p<0.01$ or 0.05 ), but there was no statistically significant difference be- tween 12-month VWF or endothelin-1 and the baseline values $(\mathrm{p}>0.05)$.

\section{Comparison of VWF and Endothelin-1 between}

Restenosis and Non-Restenosis Groups

As shown in table 3, there was no statistically significant difference in the baseline levels of VWF or endothelin-1 between restenosis and non-restenosis groups ( $p>$ $0.05)$. In both groups, the 1-hour VWF and endothelin-1 were higher than the baseline values $(\mathrm{p}<0.05)$. In the nonrestenosis group, the mean VWF and endothelin-1 levels at 2 weeks, 1, 6 and 12 months after CAS were similar to the baseline values. In the restenosis group, the mean VWF and endothelin-1 levels at 2 weeks, 1 and 6 months after CAS were higher than the baseline levels ( $p<0.05$ or $p<0.01$ ). The mean levels of VWF and endothelin- 1 in the restenosis group were also higher than in the non-restenosis group at $1 \mathrm{~h}, 2$ weeks, 1 and 6 months after CAS $(\mathrm{p}<0.05$ or $\mathrm{p}<0.01)$. There was no statistically significant difference in 12-month VWF or endothelin-1 between restenosis and non-restenosis groups ( $\mathrm{p}>0.05)$.

\section{Discussion}

In the present study, $23 \%$ of the patients showed some evidence of in-stent restenosis; the rate of significant restenosis $(>50 \%)$ was only $4.9 \%$. Of all patients who had reste- 
nosis, there were no significant ischemic symptoms to suggest further interventions were required. These results indicate that with optimal use of medical therapies to control cardiovascular risk factors such as cholesterol, hypertension or diabetes [10], the incidence of in-stent restenosis was low within the first 12 months of CAS. Our results are consistent with the previous reports $[4,5,11-13]$ that CAS appears to be clinically safe and is associated with a low rate of periprocedural and 1-year cerebrovascular events or mortality. The reported incidence of in-stent restenosis following CAS ranged from 4.5 to $33.3 \%$ [14-18]. Unlike in the present study where CT carotid angiogram was used to diagnose post-CAS restenosis, duplex ultrasonography was the most commonly used technique to detect the post-CAS in-stent restenosis $[14,19]$. However, the ultrasound criteria for determining a restenosis are very heterogeneous [20], and whether CT angiography is a better follow-up modality than ultrasound requires further investigation.

There has been limited information about the pathogenesis and risk factors for in-stent restenosis following CAS. Previous studies [20-22] suggested that advanced age, female gender, implantation of multiple stents, prior stroke or revascularization treatment, suboptimal result with residual stenosis, and elevated postprocedural serum levels of acute-phase reactants may be associated with restenosis [20-22]. VWF and endothelin-1 are endothelium injury markers and are valuable for assessing the risk of thrombogenesis [6-8]. VWF contributes to thrombogenesis by mediating cluster and adhesion of platelets at the site of vascular injury [23]. Endothelin-1 is a major vasoconstrictor, and an increased release of endothelin-1 promotes vascular smooth muscle cell proliferation and accelerates the thrombogenesis [24]. The endothelin-1 receptor system has been known to play an important role in the pathogenesis of neointimal hyperplasia after endothelial injury [24]. Therefore antagonism of the endothelin receptor system may be effective in preventing restenosis after angioplasty. In the present study, there was an increase in plasma VWF and endothelin-1 in the first hour following CAS in all patients. In the non-restenosis group, plasma VWF and endothelin-1 levels returned to baseline levels at week 2 . In the restenosis group, plasma VWF and endothelin-1 remained higher than the baseline levels until 6 months after CAS. The mean values of VWF and endothelin-1 in the first 6 months following CAS were higher than in the non-restenosis group. These results suggest that persistent elevation in plasma VWF and endothelin-1 within the first 6 months of CAS may be associated with in-stent restenosis.

Considerable controversy exists regarding the clinical significance, natural history, threshold for management, and appropriate intervention of recurrent carotid stenosis after CAS [25]. Similar to the results from our study, many patients with post-CAS restenosis remain asymptomatic and probably do not require second revascularization procedures [20]. However, patients with symptomatic and severe restenosis may require further assessment and intervention [25]. There has been little information about the strategies in preventing in-stent restenosis. Given the established role of diabetes, smoking, dyslipidemia or hypertension in the pathogenesis of carotid artery stenosis, optimal control of these cardiovascular risk factors may reduce the risk of restenosis following CAS. Further studies are required to ascertain if endothelium protection measures may reduce or prevent post-CAS restenosis.

More than $85 \%$ of our patients were treated with an angiotensin-converting enzyme inhibitor or an angiotensin receptor blocker following CAS. Previous experimental studies had shown that angiotensin-converting enzyme inhibitors or angiotensin receptor blockers inhibit plasma or vascular tissue levels of endothelin-1 [26], and some angiotensin receptor blockers also block vascular endothelin receptors [27]. However, in healthy subjects and in normotensive type 1 diabetic patients, angiotensin-converting enzyme inhibitors had no impact on the plasma levels of VWF or endothelin-1 [28]. In the present study, the proportion of patients who were treated with an angiotensin-converting enzyme inhibitor or an angiotensin receptor blocker was similar between the restenosis and non-restenosis groups (85.7 vs. $87.2 \%$ ). Therefore, these agents were unlikely to have contributed to the differences in plasma VWF and endothelin-1 levels between the two groups of patients.

There are many other factors, such as hypertension, diabetes, advanced age and sex that may contribute to the restenosis following carotid stenting. Due to the relatively small number of patients in the present study, multivariate regression analysis on the risk factors for carotid restenosis was not performed. However, there was no statistically significant difference in the proportion of male patients, mean age, and incidence of diabetes or hypertension between the restenosis and non-restenosis groups. Therefore, these factors were unlikely to be the independent predictors of restenosis following carotid stenting in our patients.

A major limitation of the present study was the relatively small patient sample. As a result, a meaningful cutoff value of VWF or endothelin-1 for post-CAS restenosis prediction could not be determined, as the number of patients with severe carotid restenosis was very small ( $\mathrm{n}=$ 3). Therefore, a larger prospective clinical trial is warranted to ascertain the predictive value of VWF or endothelin-1 for post-CAS restenosis. 


\section{Conclusion}

This prospective study demonstrated that VWF and endothelin-1 were elevated immediately after CAS. Persistent elevation of the two biomarkers was found in patients with in-stent restenosis following CAS.

\section{Acknowledgment}

This study was funded by the Chinese Ministry of Science.

\section{References}

1 Volcik KA, Ballantyne CM, Coresh J, Folsom AR, Wu KK, Boerwinkle E: P-selectin Thr 715 Pro polymorphism predicts P-selectin levels but not risks of incident coronary heart disease or ischemic stroke in a cohort of 14,595 participants: the Atherosclerosis Risk in Communities Study. Atherosclerosis 2006; 186:74-79.

-2 Lanzino G, Tallarita T, Rabinstein AA: Internal carotid artery stenosis: natural history and management. Semin Neurol 2010;30: 518-527.

-3 De Weerd M, Greving JP, Hedblad B, Lorenz MW, Mathiesen EB, O'Leary DH, Rosvall M, Sitzer M, Buskens E, Bots ML: Prevalence of asymptomatic carotid artery stenosis in the general population: an individual participant data meta-analysis. Stroke 2010;41: 1294-127.

4 Lal BK, Kaperonis EA, Cuadra S, Kapadia I, Hobson RW 2nd: Patterns of in-stent restenosis after carotid artery stenting: classification and implications for long-term outcome. J Vasc Surg 2007;46:833-840.

-5 Ederle J, Dobson J, Featherstone RL, Bonati LH, van der Worp HB, de Borst GJ, Lo TH, Gaines P, Dorman PJ, Macdonald S, Lyrer PA, Hendriks JM, McCollum C, Nederkoorn PJ, Brown MM: Carotid artery stenting compared with endarterectomy in patients with symptomatic carotid stenosis (International Carotid Stenting Study): an interim analysis of a randomised controlled trial. Lancet 2010;375:985-997.

-6 Heper G, Murat SN, Durmaz T, Kalkan F: Prospective evaluation of von Willebrand factor release after multiple and single stenting. Angiology 2004;55:177-186.

$\rightarrow 7$ Wainstein MV, Goncalves SC, Zago AJ, Zenker R, Burttet R, Couto Gde B, Tomazi F, Ribeiro JP: Plasma endothelin-1 levels after coronary stenting in humans. Am J Cardiol 2003;92:1211-1214.

-8 Takase H, Sugiyama M, Nakazawa A, Toriyama T, Hayashi K, Goto T, Sato K, Ikeda K, Ueda R, Dohi Y: Increased endogenous endothelin-1 in coronary circulation is associated with restenosis after coronary angioplasty. Can J Cardiol 2003;19:902-906.
9 Xia ZY, Yang H, Qu HG, Cheng WD, Wang LX: Expression of P-selectin, von Willebrand and endothelin-1 after carotid artery stenting. Vasa 2011;40:199-204.

10 Ovbiagele B: Optimizing vascular risk reduction in the stroke patient with atherothrombotic disease. Med Princ Pract 2010; 19:1-12.

11 Tallarita T, Oderich GS, Lanzino G, Cloft H, Kallmes D, Bower TC, Duncan AA, Gloviczki P: Outcomes of carotid artery stenting versus historical surgical controls for radiation-induced carotid stenosis. J Vasc Surg 2011;53:629-636

12 Marone EM, Coppi G, Tshomba Y, Chiesa R: Eight-year experience with carotid artery stenting for correction of symptomatic and asymptomatic post-endarterectomy defects. J Vasc Surg 2010;52:1511-1517.

13 White RA, Sicard GA, Zwolak RM, Sidawy AN, Schermerhorn ML, Shackelton RJ, Siami FS, SVS Outcomes Committee: Society of Vascular Surgery vascular registry comparison of carotid artery stenting outcomes for atherosclerotic versus nonatherosclerotic carotid artery disease. J Vasc Surg 2010;51: 1116-1123.

14 Cumbie T, Rosero EB, Valentine RJ, Modrall JG, Clagett GP, Timaran CH: Utility and accuracy of duplex ultrasonography in evaluating in-stent restenosis after carotid stenting. Am J Surg 2008;196:623-628.

15 Takigawa T, Matsumaru Y, Hayakawa M, Nemoto S, Matsumura A: Cilostazol reduces restenosis after carotid artery stenting. J Vasc Surg 2010;51:51-56.

16 Maleux G, Marrannes J, Heye S, Daenens K, Verhamme P, Thijs V: Outcome of carotid artery stenting at 2 years follow-up: comparison of nitinol open cell versus stainless steel closed cell stent design. J Cardiovasc Surg 2009;50:669-675.

17 Harrer JU, Morschel R, Mull M, Kosinski CM: High rate of restenosis after carotid artery stenting in patients with high-grade internal carotid artery stenosis: medium-term follow-up. J Neurol 2008;255:1309-1314.

18 Lal BK, Kaperonis EA, Cuadra S, Kapadia I, Hobson RW 2nd: Patterns of in-stent restenosis after carotid artery stenting: classification and implications for long-term outcome. J Vasc Surg 2007;46:833-840.
19 Zhou W, Felkai DD, Evans M, McCoy SA Lin PH, Kougias P, El-Sayed HF, Lumsden $A B$ : Ultrasound criteria for severe in-stent restenosis following carotid artery stenting. J Vasc Surg 2008;47:74-80.

20 Van Laanen J, Hendriks JM, Van Sambeek MR: Factors influencing restenosis after carotid artery stenting. J Cardiovasc Surg 2008; 49:743-747.

21 Skelly CL, Gallagher K, Fairman RM, Carpenter JP, Velazquez OC, Parmer SS, Woo EY: Risk factors for restenosis after carotid artery angioplasty and stenting. J Vasc Surg 2006;44:1010-1015.

22 Clark DJ, Lessio S, O’Donoghue M, Tsalamandris C, Schainfeld R, Rosenfield K: Mechanisms and predictors of carotid artery stent restenosis: a serial intravascular ultrasound study. J Am Coll Cardiol 2006;47: 2390-2396.

23 Gailani D, Renne T: Intrinsic pathway of coagulation and arterial thrombosis. Arterioscler Thromb Vasc Biol 2007;27:2507-2513.

- 24 Kitada K, Yui N, Matsumoto C, Mori T, Ohkita M, Matsumura Y: Inhibition of endothelin ETB receptor system aggravates neointimal hyperplasia after balloon injury of rat carotid artery. J Pharmacol Exp Ther 2009; 331:998-1004.

25 Lal BK, Hobson RW 2nd: Management of carotid restenosis. J Cardiovasc Surg 2006;47: 153-160.

26 Lariviere R, Lebel M, Kingma I, Grose JH, Boucher D: Effects of losartan and captopril on endothelin-1 production in blood vessels and glomeruli of rats with reduced renal mass. Am J Hypertens 1998;11:989-997.

27 Kurtz TW, Klein U: Next generation multifunctional angiotensin receptor blockers. Hypertens Res 2009;32:826-834.

-28 Schalkwijk CG, Smulders RA, Lambert J, Donker AJ, Stehouwer CD: ACE-inhibition modulates some endothelial functions in healthy subjects and in normotensive type 1 diabetic patients. Eur J Clin Invest 2000;30: 853-860. 